\title{
$\gamma$-STRONGLY CONVEX FUNCTIONS AND $\gamma$-SUBDIFFERENTIABILITY WITH APPLICATION TO NONLINEAR PARTIAL DIFFERENTIAL EQUATIONS
}

\author{
IKRAM DAIDAI*, TIJANI AMAHROQ AND AICHA SYAM
}

\begin{abstract}
This paper introduces a new class of convex functions called $\gamma$-strongly convex functions and studies some properties of it. It also defines a new subdifferentiability called $\gamma$-subdifferentiability and investigate some properties of it. A characterization of lower semicontinuous $\gamma$-strongly convex functions in Banach spaces is shown. Then we remove to present a result concerning optimization problems for $\gamma$-strongly convex functions which allows us to conclude an extension of Minty-Browder and nonlinear Lax-Milgram theorems in Banach spaces. Finally an example of nonlinear partial differential equations is provided.
\end{abstract}

\section{INTRODUCTION.}

Let $(V,\|\|)$ be a Banach space and $V^{*}$ its topological dual space and let $f: V \rightarrow \mathbb{R} \cup\{+\infty\}$ be an extended-real-valued function. It is well known that convex functions are the most useful instruments responsible for the success of analysis. Due to the concept of convexity several concepts and results have been introduced such as the concept of Fenchel subdifferential which is expressed at a point $x$ where $f$ is finite as follows

$$
\partial f(x)=\left\{x^{*} \in V^{*},<x^{*}, y-x>\leq f(y)-f(x), \forall y \in V\right\} .
$$

A number of results and rules have been proved. In particular it has been shown that a l.s.c. function defined on a Banach space is convex iff its Clarke subdifferential is monotone setvalued map. The first work has been done by A. Poliquin [15] who has worked in the finite dimensional case and this fact had been remarked by F. Clarke [7] for a locally Lipschitzian function in Banach space. In [8] R. Correa, A. Jofré and L. Thibault proved the same result on reflexive Banach space by using Moreau-Yosida proximal approximation. In [9] the result was extended in Banach space by using the mean value theorem of D. Zagrodny [24] and L. Thibault [23].

In connection with the concept of convex functions many other types of functions have been studied as well as the concept of c-convex functions which have been considered by T. Polyak [16] and the concept of $\gamma$-paraconvex functions and maps introduced by S. Rolewicz [20-22] and studied by A. Jourani [11], A. Allali and T. Amahroq [2], T. Amahroq and A. Taa [3] and other

Faculté des Sciences et Techniques, Université Cadi Ayyad, B.P. 549, Marrakech, Morocco

*Corresponding author

E-mail addresses: ikram_daidai_57@yahoo.fr, amahroq@gmail.com, aicha.syam@gmail.com.

Key words and phrases. $\gamma$-strong convexity; $\gamma$-strong monotonicity; $\gamma$-subdifferentiability; Minty-Browder theorem; nonlinear Lax-Milgram theorem; p-Laplacian.

Received 09/08/2021. 
authors.

In this paper we introduce a new class of convex functions that will be called $\gamma$-strongly convex functions and give some characterizations of it. A new subdifferential is also presented, this alows us to give another characterization of such functions in terms of $\gamma$-monotonicity of its presubdifferential. This fact deduces the result obtained by R. Correa, A. Jofré and L. Thibault $[8,9]$.

In the last section we establish an important tool to solve unconstrained optimization problem for $\gamma$-strongly convex functions in Banach spaces. This result turns out to be an extension of Minty-Browder theorem in Banach spaces and hence finds out Lax-Milgram theorem. Our results are stronger than the classical ones even in the linear case. We end this paper by applying our results to p-Laplace problem.

\section{PRELIMINARIES.}

Throughout this paper $V$ is a Banach space, $V^{*}$ its topological dual and $\langle,>$ the bilinear form of duality. We denote by $\|$. $\|$ the norm in $V,\|.\|_{*}$ the norm in $V^{*}$ and $\mathbb{B}(x, r)$ the open ball centered at $x$ with radius $r$ in $V$.

In what follows we recall some notions and results from nonsmooth analysis. It is well known that for any convex function $f: V \rightarrow \mathbb{R} \cup\{+\infty\}$, the "right hand" directional derivative at $x$ in $\operatorname{dom} f$ (the domain of $f$ ) exists and is for each $h \in V$

$$
d^{+} f(x)(h)=\lim _{t \rightarrow 0^{+}} \frac{f(x+t h)-f(x)}{t} .
$$

The function $f$ is said to be Gâteaux-differentiable at $x \in \operatorname{dom} f$ provided the limit

$$
<f^{\prime}(x), h>=\lim _{t \rightarrow 0} \frac{f(x+t h)-f(x)}{t},
$$

exists for each $h \in V$. The function $f^{\prime}(x)$ is called the Gâteaux-derivative of $f$ at $x$. Let $f$ : $V \rightarrow \mathbb{R} \cup\{+\infty\}$ be a function. The Clarke subdifferential of $f$ at a point $x \in \operatorname{dom} f$ is defined by

$$
\partial_{c} f(x)=\left\{x^{*} \in V^{*}, \quad<x^{*}, h>\leq f^{o}(x ; h), \forall h \in V\right\}
$$

where

$$
f^{o}(x ; h)=\limsup _{\substack{u \rightarrow f \\ t \rightarrow 0^{+}}} \inf _{y \rightarrow h} \frac{f(u+t y)-f(u)}{t} .
$$

For the definition of limsup inf and the properties of $\partial_{c} f$ and $f^{o}$ see Rockafellar $[18,19]$. When $f$ is convex, then $\partial_{c} f(x)$ coincides with the Fenchel subdifferential (see [7]), that is

$$
\partial f(x)=\left\{x^{*} \in V^{*}:<x^{*}, y-x>\leq f(y)-f(x), \forall y \in V\right\} .
$$

We extend these definitions by setting $\partial f(x)=\partial_{c} f(x)=\emptyset$ if $x \notin \operatorname{dom} f$. Moreover if $f$ is continuous at a point $x \in \operatorname{dom} f$ then $\partial f(x) \neq \emptyset$.

For any $\varepsilon>0$, the $\varepsilon$-subdifferential of $f$ at a point $x \in d o m f$ is the set defined by

$$
\partial_{\varepsilon} f(x)=\left\{x^{*} \in V^{*}:<x^{*}, y-x>\leq f(y)-f(x)+\varepsilon, \forall y \in V\right\} .
$$


And $\partial_{\varepsilon} f(x)=\emptyset$ when $x \notin d o m f$. Clearly $\partial_{\varepsilon_{1}} f(x) \subset \partial_{\varepsilon_{2}} f(x)$ whenever $\varepsilon_{1} \leq \varepsilon_{2}$. It is well known [14] that if $f$ is proper, l.s.c. and convex, then for each $\varepsilon>0$ and $x \in \operatorname{dom} f$, the set $\partial_{\varepsilon} f(x)$ is nonempty. Under the same hypotheses we have

$$
d^{+} f(x)(h)=\lim _{\varepsilon \rightarrow 0^{+}} \sup \left\{<x^{*}, h>, x^{*} \in \partial_{\varepsilon} f(x)\right\}, \quad \forall h \in V .
$$

We also recall that a set-valued map $F: V \rightrightarrows V^{*}$ is monotone iff for all $x, y \in V, x^{*} \in F(x)$ and $y^{*} \in F(y)$ we have

$$
<x^{*}-y^{*}, x-y>\geq 0 .
$$

And we write $d o m F=\{x \in V, F(x) \neq \emptyset\}$ for its domain.

\section{The $\gamma$-STRongly COnvex Functions.}

In this section we bring into light a class of functions that are strongly convex that will be called $\gamma$-strongly convex functions.

Definition 1.1. Let $f: V \rightarrow \mathbb{R} \cup\{+\infty\}$ be a function and $\gamma$ be a real positive number $(\gamma>0)$. We will say that $f$ is $(\gamma, c)$-convex if there exist a constant $c \geq 0$ and a function $g$ : $[0,1] \rightarrow \mathbb{R}^{+}$with

$$
\lim _{\theta \rightarrow 0} \frac{g(\theta)}{\theta}<+\infty \quad \text { and } \quad g(0)=g(1)=0
$$

such that for all $x, y \in V$ and $\theta \in[0,1]$

$$
f(\theta y+(1-\theta) x) \leq \theta f(y)+(1-\theta) f(x)-c g(\theta)\|x-y\|^{\gamma} .
$$

If $c>0$, the function is said to be $\gamma$-strongly convex.

If $c=0$, the function is convex.

Remark 1.2. Note that when $g(\theta)=\frac{\theta(1-\theta)}{2}$ and $\gamma=2$ we obtain the functions called stronglyconvex.

The following proposition is an obvious consequence.

Proposition 1.3. i) If $f_{1}$ and $f_{2}$ are respectively $\left(\gamma, c_{1}\right)$-convex and $\left(\gamma, c_{2}\right)$-convex, and $\alpha_{1}$ and $\alpha_{2}$ are positive real numbers, then $\alpha_{1} f_{1}+\alpha_{2} f_{2}$ is $\left(\gamma, \alpha_{1} c_{1}+\alpha_{2} c_{2}\right)$-convex.

ii)If the functions $f_{i}, i \in\{1, \ldots, n\}$ are respectively $\left(\gamma, c_{i}\right)$-convex, then the function $f=\sup _{i \in I} f_{i}$ is $(\gamma, c)-$ convex with $c=\inf _{i \in I} c_{i}$.

The following proposition shows an equivalent definition of $\gamma$-strong convexity when a function is l.s.c.

Proposition 1.4. Let $f: V \rightarrow \mathbb{R} \cup\{+\infty\}$ be a l.s.c. function. Then $f$ is $\gamma$-strongly convex with $\gamma>0$ iff there exists $c>0$ such that for all $x, y \in V$

$$
f\left(\frac{x+y}{2}\right) \leq \frac{1}{2} f(x)+\frac{1}{2} f(y)-c\|x-y\|^{\gamma} .
$$

Proof. We easily see that (1.1) implies (1.2), it suffices to set $\theta=\frac{1}{2}$ in (1.1). Conversely, suppose that (1.2) holds and fix $x, y \in V$. For $n \geq 1$ we show by induction on $n$ the following property

$$
\left(P_{n}\right) \quad f(\theta x+(1-\theta) y) \leq \theta f(x)+(1-\theta) f(y)-\frac{c}{2}\|x-y\|^{\gamma}, \quad \forall \theta \in \widetilde{K_{n}},
$$


where

$$
\widetilde{K_{n}}=\{\theta \in] 0,1\left[, \quad 2^{n} \theta \in \mathbb{N}\right\} .
$$

If $n=1$, then $\left(P_{1}\right)$ reduces to (1.2). Assuming $\left(P_{n}\right)$ to hold for some $n \in \mathbb{N}$, we will prove it for $n+1$.

Let $\theta \in \widetilde{K_{n+1}} \backslash \widetilde{K_{n}}\left(\right.$ since $\left.\widetilde{K_{n}} \subset \widetilde{K_{n+1}}\right)$, then there exist $\theta_{1}, \theta_{2} \in \widetilde{K_{n}} \cup\{0,1\}$ such that $\theta=\frac{\theta_{1}+\theta_{2}}{2}$ with $\theta_{1}<\theta_{2}$. It follows by (1.2)

$$
f(\theta x+(1-\theta) y) \leq \frac{1}{2} f\left(\theta_{1} x+\left(1-\theta_{1}\right) y\right)+\frac{1}{2} f\left(\theta_{2} x+\left(1-\theta_{2}\right) y\right)-c\left(\theta_{2}-\theta_{1}\right)^{\gamma}\|x-y\|^{\gamma} .
$$

Using the induction assumption, we get

$$
f(\theta x+(1-\theta) y) \leq \theta f(x)+(1-\theta) f(y)-\frac{c}{2}\|x-y\|^{\gamma} .
$$

What means that $\left(P_{n+1}\right)$ is verified and then $\left(P_{n}\right)$ holds for all $n \in \mathbb{N}$. So

$$
f(\theta x+(1-\theta) y) \leq \theta f(x)+(1-\theta) f(y)-\frac{c}{2}\|x-y\|^{\gamma}, \quad \forall \theta \in \cup_{n} \widetilde{K_{n}} .
$$

Since $\min (\theta, 1-\theta) \leq \frac{1}{2}$, then

$$
f(\theta x+(1-\theta) y) \leq \theta f(x)+(1-\theta) f(y)-c \min (\theta, 1-\theta)\|x-y\|^{\gamma}, \quad \forall \theta \in \underset{n \geq 0}{\cup} K_{n},
$$

where

$$
K_{n}=\left\{\theta \in[0,1], \quad 2^{n} \theta \in \mathbb{N}\right\} .
$$

Now let $\theta \in[0,1]$. Since $[0,1]=\overline{\bigcup_{n \geq 0} K_{n}}$, then there exists $\left(\theta_{n}\right) \in \underset{n \geq 0}{\cup} K_{n}$ such that $\theta_{n} \rightarrow \theta$ and so

$$
f\left(\theta_{n} x+\left(1-\theta_{n}\right) y\right) \leq \theta_{n} f(x)+\left(1-\theta_{n}\right) f(y)-c \min \left(\theta_{n}, 1-\theta_{n}\right)\|x-y\|^{\gamma} .
$$

However $f$ is l.s.c., thus

$$
f(\theta x+(1-\theta) y) \leq \theta f(x)+(1-\theta) f(y)-c g(\theta)\|x-y\|^{\gamma},
$$

where $g(\theta)=\min (\theta, 1-\theta)$. Since $\theta \in[0,1]$ and $x, y \in V$ are arbitrary and the fact that $g(0)=g(1)=0$ and $\lim _{\theta \rightarrow 0} \frac{g(\theta)}{\theta}<+\infty$, we deduce that $f$ is $\gamma$-strongly convex.

When $f$ is Gâteaux-differentiable function we get the following result.

Proposition 1.5. Let $f: V \rightarrow \mathbb{R} \cup\{+\infty\}$ be a Gâteaux-differentiable function and $\gamma \geq 1$. Then the following assertions are equivalent

i): $f$ is $\gamma$-strongly convex,

ii): $\left\langle f^{\prime}(x), y-x>\leq f(y)-f(x)-\frac{c}{\gamma}\|x-y\|^{\gamma}, \quad \forall x, y \in V\right.$.

Proof. $i) \Rightarrow i i)$ Let $x, y \in V$ be fixed. Since $f$ is $\gamma$-strongly convex then there exist $c>0$ and $g:[0,1] \rightarrow \mathbb{R}^{+}$with $\lim _{\theta \rightarrow 0} \frac{g(\theta)}{\theta}<+\infty$ and $g(0)=g(1)=0$, such that

$$
f(\theta y+(1-\theta) x) \leq \theta f(y)+(1-\theta) f(x)-c g(\theta)\|x-y\|^{\gamma} .
$$

We may assume without loss of generality that $\lim _{\theta \rightarrow 0} \frac{g(\theta)}{\theta}=1$. Dividing by $\left.\left.\theta \in\right] 0,1\right]$ and passing to the limit as $\theta \rightarrow 0$, we obtain for all $x, y \in V^{\theta \rightarrow}$

$$
<f^{\prime}(x), y-x>\leq f(y)-f(x)-\frac{c}{\gamma}\|x-y\|^{\gamma} .
$$

$i i) \Rightarrow i)$ We have for all $x, y \in V$ and all $\theta \in[0,1]$

$$
-\theta<f^{\prime}(\theta y+(1-\theta) x), y-x>\leq f(x)-f(\theta y+(1-\theta) x)-\frac{c}{\gamma} \theta^{\gamma}\|x-y\|^{\gamma},
$$




$$
(1-\theta)<f^{\prime}(\theta y+(1-\theta) x), y-x>\leq f(y)-f(\theta y+(1-\theta) x)-\frac{c}{\gamma}(1-\theta)^{\gamma}\|x-y\|^{\gamma} .
$$

And so

$$
\begin{gathered}
-(1-\theta) \theta<f^{\prime}(\theta y+(1-\theta) x), y-x>\leq(1-\theta) f(x)-(1-\theta) f(\theta y+(1-\theta) x)-\frac{c}{\gamma}(1-\theta) \theta^{\gamma}\|x-y\|^{\gamma}, \\
\theta(1-\theta)<f^{\prime}(\theta y+(1-\theta) x), y-x>\leq \theta f(y)-\theta f(\theta y+(1-\theta) x)-\frac{c}{\gamma} \theta(1-\theta)^{\gamma}\|x-y\|^{\gamma} .
\end{gathered}
$$

Then

$$
f(\theta y+(1-\theta) x) \leq \theta f(y)+(1-\theta) f(x)-c g(\theta)\|x-y\|^{\gamma},
$$

with $g(\theta)=\frac{1}{\gamma}\left((1-\theta) \theta^{\gamma}+\theta(1-\theta)^{\gamma}\right)$, since $\gamma \geq 1$ then $\lim _{\theta \rightarrow 0} \frac{g(\theta)}{\theta}<+\infty$ and $g(0)=g(1)=0$. Which completes the proof.

\section{The $\gamma$-SUBDifFEREnTIABILITY.}

In this section we highlight a new subdifferentiability that will be called the $\gamma$-subdifferentiability. When $f$ is convex we show that this subdifferential is smaller than the Fenchel subdifferential.

Definition 2.1. Let $f: V \rightarrow \mathbb{R} \cup\{+\infty\}$ be an extended real-valued function, $x \in \operatorname{dom} f$, $\gamma>0$ and $c>0$. We will say that $x^{*} \in V^{*}$ is local $(\gamma, c)$-subgradient of $\mathrm{f}$ at $\mathrm{x}$ if there exists $\delta>0$ such that

$$
<x^{*}, y-x>\leq f(y)-f(x)-\frac{c}{\gamma}\|y-x\|^{\gamma}, \forall y \in \mathbb{B}(x, \delta) .
$$

The set of all local $(\gamma, c)$-subgradients of $\mathrm{f}$ at $\mathrm{x}$ will be denoted by $\partial_{(\gamma, c)}^{\text {loc }} f(x)$. When $x \notin d o m f$ then $\partial_{(\gamma, c)}^{l o c} f(x)=\emptyset$. This set can be expressed globally in the following sense, for all $x \in \operatorname{dom} f$

$$
\partial_{(\gamma, c)} f(x)=\left\{x^{*} \in V^{*}, \quad<x^{*}, y-x>\leq f(y)-f(x)-\frac{c}{\gamma}\|y-x\|^{\gamma}, \forall y \in V\right\},
$$

and $\partial_{(\gamma, c)} f(x)=\emptyset$ when $x \notin d o m f$.

Remark 2.2. a)It is clear that the set of local $(\gamma, c)$-subgradients at $x$ is convex and weak-star closed.

b)If the function $\varphi:=f-\frac{c}{\gamma}\|x-\|^{\gamma}$ is convex then it is easy to check that $x^{*} \in \partial \varphi(x)$ iff $x^{*} \in \partial_{(\gamma, c)} f(x)$.

Example 2.3. As an example we consider the real function $f(x):=|x|$. It is easy to show that $\partial_{(1, c)} f(0)=[c-1,1-c]$ when $c \leq 1$ and $\partial_{(1, c)} f(0)=\emptyset$ when $c>1$.

The following proposition shows that when $f$ is convex, the set $\partial_{(\gamma, c)}^{l o c} f$ is smaller than the Fenchel subdifferential.

Proposition 2.4. Let $f: V \rightarrow \mathbb{R} \cup\{+\infty\}$ be an extended real-valued function, proper and convex, $\gamma>0$ and $c>0$. Then for any $x \in \operatorname{dom} f$ one has

$$
\partial_{(\gamma, c)}^{l o c} f(x) \subset \partial f(x) .
$$

Proof. Let $x \in \operatorname{dom} f$ and $x^{*} \in \partial_{(\gamma, c)}^{l o c} f(x)$, then there exists a real positive number $\delta>0$ such that

So

$$
<x^{*}, y-x>\leq f(y)-f(x)-\frac{c}{\gamma}\|y-x\|^{\gamma}, \forall y \in \mathbb{B}(x, \delta)
$$

$$
<x^{*}, y-x>\leq f(y)-f(x), \forall y \in \mathbb{B}(x, \delta) .
$$


Now if $y \notin \mathbb{B}(x, \delta)$. Let $z=x+\theta(y-x)$ with $\theta<\frac{\delta}{\|y-x\|}$. So $z \in \mathbb{B}(x, \delta)$ and hence

$$
<x^{*}, z-x>\leq f(z)-f(x) .
$$

It follows by the convexity of $f$ that

$$
<x^{*}, y-x>\leq f(y)-f(x),
$$

which implies that $x^{*} \in \partial f(x)$.

The following example shows that in general the inclusion of Proposition 2.4 is strict.

Example 2.5. Let $V=\mathbb{R}$ and let $f$ be given by $f(x)=|x|$. We have $\partial f(0)=[-1,1]$ while $1 \notin \partial_{(\gamma, c)}^{\text {loc }} f(0)$. Indeed if $1 \in \partial_{(\gamma, c)}^{\text {loc }} f(0)$ then there exists $\delta>0$ such that

$$
\left.y \leq|y|-c|y|^{\gamma}, \forall y \in\right]-\delta, \delta[.
$$

Let $y=\frac{\delta}{2}$ then $\delta \leq 0$ which is not true.

Proposition 2.6. Let $f: V \rightarrow \mathbb{R} \cup\{+\infty\}$ be an extended real-valued function, proper and $\gamma$-strongly convex with $\gamma>1$ and $c>0$. Then

$$
\partial_{(\gamma, c)}^{l o c} f(x)=\partial_{(\gamma, c)} f(x), \forall x \in V .
$$

Proof. Obviously $\partial_{(\gamma, c)} f(x) \subset \partial_{(\gamma, c)}^{\text {loc }} f(x)$. Conversely let $x \in \operatorname{dom} f$ and $x^{*} \in \partial_{(\gamma, c)}^{l o c} f(x)$. So there exists $\delta>0$ such that

$$
<x^{*}, y-x>\leq f(y)-f(x)-\frac{c}{\gamma}\|y-x\|^{\gamma}, \forall y \in \mathbb{B}(x, \delta) .
$$

Now let us assume that $y \notin \mathbb{B}(x, \delta)$ and let $z=x+\theta(y-x)$ with $0<\theta<\frac{\delta}{\|y-x\|}$. Obviously $z \in \mathbb{B}(x, \delta)$, thus

$$
<x^{*}, z-x>\leq f(z)-f(x)-\frac{c}{\gamma}\|z-x\|^{\gamma} .
$$

Since $f$ is $\gamma$-strongly convex, we obtain

$$
<x^{*}, \theta(y-x)>\leq \theta(f(y)-f(x))-\frac{c}{\gamma} \theta^{\gamma}\|y-x\|^{\gamma}-c g(\theta)\|y-x\|^{\gamma} .
$$

Dividing by $\theta$ and passing to the limit as $\theta \rightarrow 0$, we obtain

$$
<x^{*}, y-x>\leq f(y)-f(x)-c\|y-x\|^{\gamma} .
$$

What means that $x^{*} \in \partial_{(\gamma, c)} f(x)$.

Remark 2.7. When $\gamma=1$ and $f$ is only convex, we show easily that for all $x \in V, \partial_{(1, c)}^{l o c} f(x)=$ $\partial_{(1, c)} f(x)$.

Proposition 2.8. Let $\gamma \geq 1$ and let $f: V \rightarrow \mathbb{R} \cup\{+\infty\}$ be an extended real-valued function and proper. If $\operatorname{dom}_{(\gamma, c)} f=V$ then $f$ is $\gamma$-strongly convex.

Proof. Let $x, y \in d o m f$ and $\theta \in[0,1]$. Let $z=x+\theta(y-x)$ and $z^{*} \in \partial_{(\gamma, c)} f(z)$; then

$$
<z^{*}, u-z>\leq f(u)-f(z)-\frac{c}{\gamma}\|u-z\|^{\gamma}, \quad \forall u \in V .
$$

In particular we have

$$
-\theta<z^{*}, y-x>\leq f(x)-f(z)-\frac{c}{\gamma} \theta^{\gamma}\|x-y\|^{\gamma},
$$


And so

$$
(1-\theta)<z^{*}, y-x>\leq f(y)-f(z)-\frac{c}{\gamma}(1-\theta)^{\gamma}\|x-y\|^{\gamma} .
$$

Therefore

$$
\begin{gathered}
-(1-\theta) \theta<z^{*}, y-x>\leq(1-\theta) f(x)-(1-\theta) f(z)-\frac{c}{\gamma}(1-\theta) \theta^{\gamma}\|x-y\|^{\gamma}, \\
\theta(1-\theta)<z^{*}, y-x>\leq \theta f(y)-\theta f(z)-\frac{c}{\gamma} \theta(1-\theta)^{\gamma}\|x-y\|^{\gamma} .
\end{gathered}
$$

$$
f(\theta y+(1-\theta) x) \leq \theta f(y)+(1-\theta) f(x)-c g(\theta)\|x-y\|^{\gamma},
$$

with $g(\theta)=\frac{1}{\gamma}\left((1-\theta) \theta^{\gamma}+\theta(1-\theta)^{\gamma}\right)$, since $\gamma \geq 1$ then $\lim _{\theta \rightarrow 0} \frac{g(\theta)}{\theta}<+\infty$ and $g(0)=g(1)=0$. Which shows that $f$ is $\gamma$-strongly convex.

Proposition 2.9. Let $f: V \rightarrow \mathbb{R} \cup\{+\infty\}$ be an extended real-valued function and proper. Suppose that $f$ is $\gamma$-strongly convex with constant $c>0$ and $\gamma \geq 1$, then

$$
\partial_{(\gamma, c)} f(x)=\partial f(x), \quad \forall x \in V .
$$

Proof. Let $x, y \in d o m f$. Clearly $\partial_{(\gamma, c)} f(x) \subset \partial f(x)$. Now let $x^{*} \in \partial f(x)$ then for all $z \in V$

$$
<x^{*}, z-x>\leq f(z)-f(x) .
$$

Let $z=x+\theta(y-x)$ with $\theta \in] 0,1[$, we obtain by $\gamma$-strong convexity of $f$

$$
\theta<x^{*}, y-x>\leq \theta(f(y)-f(x))-c g(\theta)\|y-x\|^{\gamma} .
$$

We may assume without loss of generality that $\lim _{\theta \rightarrow 0} \frac{g(\theta)}{\theta}=1$. Dividing by $\left.\left.\theta \in\right] 0,1\right]$ and passing to the limit as $\theta \rightarrow 0$, we get $x^{*} \in \partial_{(\gamma, c)} f(x)$.

In connection with the results concerning the Fenchel subdifferential, Proposition 2.9 allows us to deduce the following corollaries.

Corollary 2.10. Let $f: V \rightarrow \mathbb{R} \cup\{+\infty\}$ be an extended real-valued function, proper and $\gamma$-strongly convex with $\gamma \geq 1$ and constant $c>0$. Suppose that $f$ is continuous at $x \in$ dom $f$ then

$$
\partial_{(\gamma, c)} f(x) \neq \emptyset \text {. }
$$

Corollary 2.11. Let $f: V \rightarrow \mathbb{R} \cup\{+\infty\}$ be an extended real-valued function, proper and $\gamma$-strongly convex with $\gamma \geq 1$. Then $f$ is Gâteaux differentiable at a point $x \in$ domf iff there exists a unique $x^{*} \in V^{*}$ such that for all $y \in V$

$$
<x^{*}, y-x>\leq f(y)-f(x)-\frac{c}{\gamma}\|y-x\|^{\gamma} .
$$

Corollary 2.12. Let $f, g: V \rightarrow \mathbb{R}$, be two proper l.s.c. and $\gamma$-strongly convex functions with constants $c_{1}$ and $c_{2}$ respectively and let $\gamma \geq 1$. Suppose that the qualification condition $(Q)$ holds,

$$
f \text { is finite and continuous at a point of dom } g .(Q)
$$

Then we have

$$
\partial_{\left(\gamma, c_{1}\right)} f+\partial_{\left(\gamma, c_{2}\right)} g=\partial_{\left(\gamma, c_{1}+c_{2}\right)}(f+g) .
$$

Proposition 2.13. Let $f: V \rightarrow \mathbb{R} \cup\{+\infty\}$ be an extended real-valued function and proper. Consider the following assertions : 
(a): $0 \in \partial_{(\gamma, c)} f(x)$.

(b): $x$ is the unique minimum of $f$.

(c): $f(y)-f(x) \geq c\|y-x\|^{\gamma}, \forall y \in V$.

Then (a) and (c) are equivalent and they imply (b). Moreover if $f$ is $(\gamma, c)$-convex then the above assertions are equivalent.

Proof. Obviously (a) is equivalent to (c) and they imply (b). Now suppose that $f$ attains a minimum at $x$, then $f(y) \geq f(x)$ for all $y \in V$. Let $y=x+\theta(z-x)$ where $\theta \in] 0,1[$ and $z \in V$, using the $\gamma$-strong convexity of $f$ we obtain

$$
f(x) \leq \theta f(z)+(1-\theta) f(x)-c g(\theta)\|z-x\|^{\gamma} .
$$

Dividing by $\theta$ and passing to the limit as $\theta \rightarrow 0$, it follows that (c) holds.

\section{Characterization of lower Semicontinuous $\gamma$-STRONGly CONVEX FunCtions.}

In this section we establish a characterization of lower semicontinuous $\gamma$-strongly convex functions. We introduce first the notion of $\gamma$-strong monotonicity and we prove that an arbitrary l.s.c. function is $\gamma$-strongly convex iff its presubdifferential map is $\gamma$-strongly monotone.

Definition 3.1. Let $\gamma>0$. A set-valued map $F: V \rightrightarrows V^{*}$ is said to be $\gamma$-strongly monotone if there exists a positive real number c such that for all $x, y \in V, x^{*} \in F(x)$ and $y^{*} \in F(y)$ one has

$$
<x^{*}-y^{*}, x-y>\geq c\|x-y\|^{\gamma} \text {. }
$$

Remark 3.2. Obviously if $\mathrm{F}$ is $\gamma$-strongly monotone, then $\mathrm{F}$ is monotone.

Remark 3.3. It is clear that the set of $(\gamma, c)$-subdifferentials is always $\gamma$-strongly monotone since we have for all $x^{*} \in \partial_{(\gamma, c)} f(x)$ and $y^{*} \in \partial_{(\gamma, c)} f(y)$

$$
<x^{*}-y^{*}, x-y>\geq \frac{2 c}{\gamma}\|x-y\|^{\gamma} .
$$

In order to prove our result for any classical subdifferential, we recall the following definition, see [23].

Definition 3.4. We call presubdifferential any operator $\tilde{\partial}$ which satisfies the following properties :

For any function $f: V \rightarrow \mathbb{R} \cup\{+\infty\}$, any continuous convex function $g: V \rightarrow \mathbb{R}$, any $\lambda>0$ and any $x \in V$

i) $\tilde{\partial} f(x) \subset V^{*}$ and $\tilde{\partial} f(x)=\emptyset$ whenever $x \notin d o m f$.

ii) $\tilde{\partial} f(x)=\partial f(x)$, whenever $f$ is convex.

iii) $\tilde{\partial}(f+\lambda g)(x) \subset \tilde{\partial} f(x)+\lambda \tilde{\partial} g(x)$.

iv) $0 \in \tilde{\partial} f(x)$ whenever $x$ is a local minimum point of $f$.

v) $\tilde{\partial} f(x)=\tilde{\partial} h(x)$ for any real-extended function $h$ which is equal to $f$ near $x$. 
Examples. Clarke's subdifferential [7], the approximate subdifferential [10,13] and others are examples of subdifferentials which verify the conditions above.

Before stating our results we need to recall the approximate mean value theorem established by Zagrodny [24] in the terms of the Clarke subdifferential which also holds for any presubdifferential satisfying the properties of Definition 3.4, see Thibault [23].

Theorem 3.5. [23, 24] Let $f: V \rightarrow \mathbb{R} \cup\{+\infty\}$ be a l.s.c. function and $a, b \in$ domf. Then there exist $c \in] a, b]$, a sequence $\left(x_{k}\right)$ converging to $c$ and $x_{k}^{*} \in \tilde{\partial} f\left(x_{k}\right)$ such that

i) $\frac{\|a-b\|}{\|a-c\|} \liminf <x_{k}^{*}, a-x_{k}>\geq f(a)-f(b)$.

ii) $\liminf <x_{k}^{*}, a-b>\geq f(a)-f(b)$.

Recall also the characterization of l.s.c. convex functions due to R. Correa, A. Jofré and L. Thibault [9] and J. M. Borwein's theorem which has a formidably long list of conclusions [5].

Theorem 3.6. [9] If $f: V \rightarrow \mathbb{R} \cup\{+\infty\}$ is l.s.c. and if $\tilde{\partial} f$ is monotone then $f$ is convex.

Theorem 3.7. [5] Let $f: V \rightarrow \mathbb{R} \cup\{+\infty\}$ be a proper convex and l.s.c. function, $\varepsilon>0$ and $\beta \geq 0$. Suppose that $x_{0} \in$ domf and $x_{0}^{*} \in \partial_{\varepsilon} f\left(x_{0}\right)$. Then there exist points $x_{\varepsilon} \in$ domf and $x_{\varepsilon}^{*} \in V^{*}$ such that
1) $x_{\varepsilon}^{*} \in \partial f\left(x_{\varepsilon}\right)$.
2) $\left\|x_{\varepsilon}-x_{0}\right\| \leq \sqrt{\varepsilon}$.
3) $x_{\varepsilon}^{*} \in \partial_{2 \varepsilon} f\left(x_{0}\right)$.
4) $\left\|x_{\varepsilon}^{*}-x_{0}^{*}\right\|_{*} \leq \sqrt{\varepsilon}\left(1+\beta\left\|x_{0}^{*}\right\|_{*}\right)$.
5) $\left|f\left(x_{\varepsilon}\right)-f\left(x_{0}\right)\right| \leq \sqrt{\varepsilon}(\sqrt{\varepsilon}+1 / \beta)$.
6) $\left|<x_{\varepsilon}^{*}-x_{0}^{*}, y>\right| \leq \sqrt{\varepsilon}\left(\|y\|+\beta\left|<x_{0}^{*}, y>\right|\right)$, for all $y \in V$.

Now, we are ready to prove the first result on the equality between the presubdifferential and $(\gamma, c)$-subdifferential.

Theorem 3.8. Let $f: V \rightarrow \mathbb{R} \cup\{+\infty\}$ be a proper and l.s.c. function and suppose that $\tilde{\partial} f$ is $\gamma$-strongly monotone, then $\partial_{(\gamma, c)} f(x)=\tilde{\partial} f(x)$ for all $x \in V$.

Proof. In view of Theorem 3.6, $f$ is in addition convex since $\tilde{\partial} f$ is $\gamma$-strongly monotone. Obviously when $x \notin d o m f$, then equality holds. Let $x, y \in d o m f$. If we set $\varphi(\theta)=f(x+\theta(y-$ $x))$ and $z_{\theta}=x+\theta(y-x)$ with $\theta \in[0,1]$, then for all $\left.s, t \in\right] 0,1[$ such that $\mathrm{s}<\mathrm{t}$ one has

$$
\varphi(t)-\varphi(s)=\int_{s}^{t} d^{+} f\left(z_{\theta}\right)(y-x) d \theta .
$$

We are justified in the equality above since $\varphi$ is continuous on $] 0,1\left[\right.$ and $d^{+} f\left(z_{\theta}\right)(y-x)$ is finite in this case. One has

$$
d^{+} f\left(z_{\theta}\right)(y-x)=\lim _{\varepsilon \rightarrow 0^{+}} \sup \left\{<x_{\varepsilon, \theta}^{*}, y-x>, x_{\varepsilon, \theta}^{*} \in \partial_{\varepsilon} f\left(z_{\theta}\right)\right\} .
$$

Let $\delta>0$ be a positive real number, then there exists a positive real number $\alpha$ with $\alpha<\delta$ such that

$$
d^{+} f\left(z_{\theta}\right)(y-x)-\delta<\sup \left\{<x_{\alpha, \theta}^{*}, y-x>, x_{\alpha, \theta}^{*} \in \partial_{\alpha} f\left(z_{\theta}\right)\right\}<d^{+} f\left(z_{\theta}\right)(y-x)+\delta .
$$

Thus for all $x_{\alpha, \theta}^{*} \in \partial_{\alpha} f\left(z_{\theta}\right)$, one has

$$
\varphi(t)-\varphi(s)>\int_{s}^{t}<x_{\alpha, \theta}^{*}, y-x>d \theta-\delta(t-s) .
$$


By applying Theorem 3.7 with $\varepsilon=\theta^{2} \alpha$ and $x_{\alpha, \theta}^{*} \in \partial_{\theta^{2} \alpha} f\left(z_{\theta}\right)$ there exist $u_{\alpha, \theta} \in$ domf and $u_{\alpha, \theta}^{*} \in V^{*}$ such that
1) $u_{\alpha, \theta}^{*} \in \partial f\left(u_{\alpha, \theta}\right)$.
2) $\left\|u_{\alpha, \theta}-z_{\theta}\right\| \leq \theta \sqrt{\alpha}$.
3) $u_{\alpha, \theta}^{*} \in \partial_{2 \theta^{2} \alpha} f\left(z_{\theta}\right)$
4) $\left\|u_{\alpha, \theta}^{*}-x_{\alpha, \theta}^{*}\right\|_{*} \leq \theta \sqrt{\alpha}\left(1+\left\|x_{\alpha, \theta}^{*}\right\|_{*}\right)$.
5) $\left|f\left(z_{\theta}\right)-f\left(u_{\alpha, \theta}\right)\right| \leq \theta \sqrt{\alpha}(\theta \sqrt{\alpha}+1)$.
6) $\left|<u_{\alpha, \theta}^{*}, u>-<x_{\alpha, \theta}^{*}, u>\right| \leq \theta \sqrt{\alpha}\left(\|u\|+\left|<x_{\alpha, \theta}^{*}, u>\right|\right), \forall u \in V$.

So

$\varphi(t)-\varphi(s)>\int_{s}^{t}<x_{\alpha, \theta}^{*}-u_{\alpha, \theta}^{*}, y-x>d \theta+\int_{s}^{t} \frac{1}{\theta}<u_{\alpha, \theta}^{*}, z_{\theta}-u_{\alpha, \theta}>d \theta+\int_{s}^{t} \frac{1}{\theta}<u_{\alpha, \theta}^{*}, u_{\alpha, \theta}-x>d \theta-\delta(t-s)$.

Let $x^{*} \in \tilde{\partial} f(x)$, then

$$
\begin{gathered}
\varphi(t)-\varphi(s)>\int_{s}^{t}<x_{\alpha, \theta}^{*}-u_{\alpha, \theta}^{*}, y-x>d \theta+\int_{s}^{t} \frac{1}{\theta}<u_{\alpha, \theta}^{*}, z_{\theta}-u_{\alpha, \theta}>d \theta+\int_{s}^{t} \frac{1}{\theta}<u_{\alpha, \theta}^{*}-x^{*}, u_{\alpha, \theta}-x>d \theta+ \\
\int_{s}^{t} \frac{1}{\theta}<x^{*}, u_{\alpha, \theta}-x>d \theta-\delta(t-s) .
\end{gathered}
$$

We get from 3) and 5) that

$$
<u_{\alpha, \theta}^{*}, z_{\theta}-u_{\alpha, \theta}>\geq-\theta \sqrt{\alpha}(\theta \sqrt{\alpha}+1)-2 \theta^{2} \alpha .
$$

It also follows from 6) that

$$
<x_{\alpha, \theta}^{*}-u_{\alpha, \theta}^{*}, y-x>>-\theta \sqrt{\alpha}\left(\|y-x\|+\left|d^{+} f\left(z_{\theta}\right)(y-x)\right|+\delta\right) .
$$

Since $\tilde{\partial} f$ is $\gamma$-strongly monotone, then

$$
\begin{gathered}
\varphi(t)-\varphi(s)>\int_{s}^{t}-\theta \sqrt{\alpha}\left(\|y-x\|+\left|d^{+} f\left(z_{\theta}\right)(y-x)\right|+\delta\right) d \theta-\int_{s}^{t} \theta(\sqrt{\alpha}(\theta \sqrt{\alpha}+1)+2 \theta \alpha) d \theta+ \\
c \int_{s}^{t} \frac{1}{\theta}\left\|u_{\alpha, \theta}-x\right\|^{\gamma} d \theta+\int_{s}^{t} \frac{1}{\theta}<x^{*}, u_{\alpha, \theta}-x>d \theta-\delta(t-s) .
\end{gathered}
$$

Taking the limit as $\delta \downarrow 0$, we obtain that $u_{\alpha, \theta} \rightarrow z_{\theta},-\sqrt{\alpha}(\theta \sqrt{\alpha}+1)-2 \theta \alpha \rightarrow 0$ and $-\theta \sqrt{\alpha}(\|$ $\left.y-x \|+\left|d^{+} f\left(z_{\theta}\right)(y-x)\right|+\delta\right) \rightarrow 0$. Since each sequence is bounded above by a constant which belongs to $L^{1}([s, t])$, we conclude by dominated convergence theorem that

$$
f(x+t(y-x)) \geq f(x+s(y-x))+(t-s)<x^{*}, y-x>+\frac{c}{\gamma}\left(t^{\gamma}-s^{\gamma}\right)\|y-x\|^{\gamma} .
$$

The inequality above holds for all $s, t \in] 0,1[\operatorname{such}$ that $s<t$. By taking the limit $s \downarrow 0$ and $t \uparrow 1$ and since $f$ is l.s.c. and convex, we get for all $x, y \in d o m f$

$$
<x^{*}, y-x>\leq f(y)-f(x)-\frac{c}{\gamma}\|y-x\|^{\gamma},
$$

which is also valid when $y \notin d o m f$. What means that $x^{*} \in \partial_{(\gamma, c)} f(x)$.

We are now in position to state our main second result. The proof has been inspired by the work of R. Correa, A. Jofré and L. Thibault [8,9].

Theorem 3.9. Let $f: V \rightarrow \mathbb{R} \cup\{+\infty\}$ be a proper and l.s.c. function. Then $\tilde{\partial} f$ is $\gamma-$ strongly monotone iff $f$ is $\gamma$-strongly convex. 
Proof. Suppose first that the presubdifferential is $\gamma$-strongly monotone. Then, Theorem 3.6 ensures that $f$ is convex. Let $x, y \in \operatorname{dom} f$, and $z=y+\theta(x-y)$ with $\theta \in] 0,1[$. As domõ $f$ is graphically dense in $\operatorname{dom} f[4,12]$, there exists a sequence $\left(y_{k}\right)$ in $\operatorname{dom} \tilde{\partial} f$ such that $y_{k} \rightarrow y$ and $f\left(y_{k}\right) \rightarrow f(y)$. Let $z_{k}=y_{k}+\theta\left(x-y_{k}\right)$. Since $f$ is convex, then $z_{k} \in \operatorname{dom} f$.

Step 1 : If $z_{k}$ is not a local minimum of $f$ we can choose a vector $z_{k}^{\prime}$ such that

$$
\left\|z_{k}^{\prime}-z_{k}\right\|<\frac{1}{k} \quad \text { and } \quad f\left(z_{k}^{\prime}\right)<f\left(z_{k}\right) .
$$

By applying Theorem 3.5 on $\left[z_{k}, z_{k}^{\prime}\right]$, we obtain a sequences $\left(z_{k, n}\right) \in V$ and $\left(z_{k, n}^{*}\right) \in V^{*}$ such that $\left.\left.z_{k, n} \underset{n}{\rightarrow} c_{k} \in\right] z_{k}, z_{k}^{\prime}\right], z_{k, n}^{*} \in \tilde{\partial} f\left(z_{k, n}\right)$, and

$$
\liminf _{n}<z_{k, n}^{*}, z_{k}-z_{k, n}>\geq\left[f\left(z_{k}\right)-f\left(z_{k}^{\prime}\right)\right] \frac{\left\|c_{k}-z_{k}\right\|}{\left\|z_{k}^{\prime}-z_{k}\right\|} \geq 0 .
$$

On the other hand we have by Theorem 3.8, $z_{k, n}^{*} \in \partial_{(\gamma, c)} f\left(z_{k, n}\right)$. Hence

$$
f(x)-f\left(z_{k, n}\right)-\frac{c}{\gamma}\left\|x-z_{k, n}\right\|^{\gamma} \geq<z_{k, n}^{*}, x-z_{k, n}>,
$$

and

$$
f\left(y_{k}\right)-f\left(z_{k, n}\right)-\frac{c}{\gamma}\left\|y_{k}-z_{k, n}\right\|^{\gamma} \geq<z_{k, n}^{*}, y_{k}-z_{k, n}>.
$$

Which implies by the lower semicontinuity of $f$ that

$$
\theta f(x)+(1-\theta) f\left(y_{k}\right)-\frac{c}{\gamma}\left((1-\theta)\left\|y_{k}-c_{k}\right\|^{\gamma}+\theta\left\|x-c_{k}\right\|^{\gamma}\right) \geq f\left(c_{k}\right) .
$$

Step 2 : If $z_{k}$ is a local minimum of $f$ then $0 \in \tilde{\partial} f\left(z_{k}\right)=\partial_{(\gamma, c)} f\left(z_{k}\right)$. Therefore setting $c_{k}=z_{k}$, we obtain

$$
f(x)-f\left(c_{k}\right)-\frac{c}{\gamma}\left\|x-c_{k}\right\|^{\gamma} \geq 0
$$

and

$$
f\left(y_{k}\right)-f\left(c_{k}\right)-\frac{c}{\gamma}\left\|y_{k}-c_{k}\right\|^{\gamma} \geq 0
$$

Which implies as in the first step that

$$
\theta f(x)+(1-\theta) f\left(y_{k}\right)-\frac{c}{\gamma}\left((1-\theta)\left\|y_{k}-c_{k}\right\|^{\gamma}+\theta\left\|x-c_{k}\right\|^{\gamma}\right) \geq f\left(c_{k}\right) .
$$

Since $f\left(y_{k}\right) \rightarrow f(y)$ and $c_{k} \rightarrow z$, we conclude by the lower semicontinuity of $f$ and the inequality above that

$$
f(\theta x+(1-\theta) y) \leq \theta f(x)+(1-\theta) f(y)-c g(\theta)\|x-y\|^{\gamma},
$$

with $\left.g(\theta)=\frac{1}{\gamma}\left(\theta(1-\theta)^{\gamma}+\theta^{\gamma}(1-\theta)\right), \theta \in\right] 0,1\left[\right.$ and $x, y \in$ domf. Since $\lim _{\theta \rightarrow 0} \frac{g(\theta)}{\theta}<+\infty$ and $g(0)=$ $g(1)=0$, we deduce that $f$ is $\gamma$-strongly convex.

The converse sense is obvious by Proposition 2.9 .

As a consequence of Theorem 3.9 is the result due to R. Correa, A. Jofré and L. Thibault [9].

Corollary 3.10. Let $f: V \rightarrow \mathbb{R} \cup\{+\infty\}$ be a proper and l.s.c. function. Then $f$ is convex iff its presubdifferential is monotone.

Proof. It suffices to take $\mathrm{c}=0$ in the Theorem 3.8 and Theorem 3.9. 
Corollary 3.11. Let $f: V \rightarrow \mathbb{R} \cup\{+\infty\}$ be a Gâteaux-differentiable function and $\gamma \geq 1$. The function $f$ is $\gamma$-strongly convex iff there exists $c>0$ such that

$$
<f^{\prime}(x)-f^{\prime}(y), x-y>\geq c\|x-y\|^{\gamma},
$$

for all $x, y \in V$.

\section{UnCONSTRAined OPtimizATION PROBLEMS IN BANACH SPACES AND NONLINEAR PDE.}

The aim of this section is to prove a result concerning a minimization problem by considering the concept of $\gamma$-strong convexity which is divided into two subsections. In the first one we prove that a l.s.c. proper and $\gamma$-strongly convex function has an unique minimum in Banach spaces. In particular our result establishes an extension of Minty-Browder theorem and hence deduces Lax-Milgram theorem in Banach spaces. In the second and last subsection we apply our theory to p-Laplace problem.

4.1. An extension of Minty-Browder theorem. Before stating our result we need to prove the following lemma. Recall that if $f: V \rightarrow \mathbb{R} \cup\{+\infty\}$ is proper, l.s.c. and convex function, then there exist $L \in V^{*}$ and $\delta \in \mathbb{R}$ such that for all $v \in V$

$$
f(v) \geq L(v)+\delta .
$$

Recall also Young's inequality that is for $a, b \geq 0$ and $\gamma>1$

$$
a b \leq \frac{1}{\gamma} a^{\gamma}+\frac{1}{\gamma^{\prime}} b^{\gamma^{\prime}}
$$

with $\frac{1}{\gamma}+\frac{1}{\gamma^{\prime}}=1$.

Lemma 4.1. Let $f: V \rightarrow \mathbb{R} \cup\{+\infty\}$ be an extended real-valued proper, l.s.c. $\gamma$-strongly convex function with $\gamma>1$, then there exist $c_{1}>0$ and $\eta \in \mathbb{R}$ such that for all $v \in V$

$$
f(v) \geq c_{1}\|v\|^{\gamma}+\eta \text {. }
$$

Proof. Let $v \in V$, we may assume without loss of generality that $0 \in \operatorname{dom} f$ then by Proposition 1.4 there exists $c>0$ such that

$$
\frac{f(v)}{2}+\frac{f(0)}{2} \geq f\left(\frac{v+0}{2}\right)+c\|v\|^{\gamma} \geq \frac{L(v)+L(0)}{2}+c\|v\|^{\gamma}+\delta .
$$

So that

$$
f(v) \geq 2 c\|v\|^{\gamma}+2 \delta-f(0)-\|L\|_{*}\|v\| .
$$

Using Young's inequality we get

$$
f(v) \geq 2 c\|v\|^{\gamma}+2 \delta-f(0)-\frac{\varepsilon^{-\gamma^{\prime}}}{\gamma^{\prime}}\|L\|_{*}^{\gamma^{\prime}}-\frac{\varepsilon^{\gamma}}{\gamma}\|v\|^{\gamma} \text {, with } \varepsilon>0 .
$$

By setting $\eta=2 \delta-f(0)-\frac{\varepsilon^{-\gamma^{\prime}}}{\gamma^{\prime}}\|L\|_{*}^{\gamma^{\prime}}$ and $c_{1}=2 c-\frac{\varepsilon^{\gamma}}{\gamma}$. By an adequate value of $\varepsilon>0$ we finish the proof.

Theorem 4.2. Let $f: V \rightarrow \mathbb{R} \cup\{+\infty\}$ be an extended real-valued proper, l.s.c. and $\gamma$-strongly convex function with $\gamma>1$. Then there exists a unique minimum $u \in V$ of $f$. Moreover there exists $c_{1}>0$ such that for all $v \in V$

$$
\|u-v\|^{\gamma} \leq c_{1}(f(v)-f(u)) .
$$


Proof. Let $\left\{u_{n}\right\}$ be a minimizing sequence of $f$ on V. Since $f$ is bounded from above, using Proposition 1.4, we get for all $n, m \in \mathbb{N}$

$$
c\left\|u_{n}-u_{m}\right\|^{\gamma} \leq \frac{1}{2}\left(f\left(u_{n}\right)-\inf _{v \in V} f(v)\right)+\frac{1}{2}\left(f\left(u_{m}\right)-\inf _{v \in V} f(v)\right) .
$$

It follows that $\left\{u_{n}\right\}$ is Cauchy sequence and hence converges to a point $u \in V$. Since $f$ is l.s.c., $u$ is a minimum of $f$ and, because of the strong convexity of $f$, this minimum is unique.

Due to Proposition 1.4 again, we have for all $v \in V$

$$
c\|u-v\|^{\gamma} \leq \frac{1}{2} f(u)+\frac{1}{2} f(v)-f\left(\frac{u+v}{2}\right) \leq \frac{f(v)-f(u)}{2} .
$$

Which finishes the proof.

The following result is an extension of Minty-Browder theorem in Banach spaces for a smaller class of monotone operators. It can be also viewed when $\gamma=2$ as an extension of nonlinear Lax-Milgram theorem.

Theorem 4.3. Let $V$ be a Banach space and let $A: V \rightarrow V^{*}$ be an operator not necessarily linear satisfying for all $u, v \in V$

$$
<A u-A v, u-v>\geq c\|u-v\|^{\gamma}
$$

for some $c>0$ and with $\gamma>1$. Let $J: V \rightarrow \mathbb{R} \cup\{+\infty\}$ be an extended real-valued proper and Gâteaux differentiable function such that $J^{\prime}(u)=A u-L$ for all $u \in V$ where $L \in V^{*}$. Then there is an unique $\bar{u} \in V$ such that $A \bar{u}=L$.

Proof. Since for all $u, v \in V<J^{\prime}(u)-J^{\prime}(v), u-v>\geq c\|u-v\|^{\gamma}$, then by Proposition $3.11 J$ is $\gamma$-strongly convex. Using Theorem 4.2 we get an unique point $\bar{u} \in V$ satisfying $A \bar{u}=L$.

In fact Theorem 4.3 establish a generalized Lax-Milgram theorem in the setting of a Banach space.

Corollary 4.4. Let $a(u, v)$ be a bilinear form on $V$ (Banach space) such that

(i): a is continuous ( i.e. for some $M>0|a(u, v)| \leq M\|u\|\|v\| \forall u, v \in V$ ).

(ii): a is coercive (i.e. for some $\alpha>0 a(u, u) \geq \alpha\|u\|^{2} \forall u \in V$ ).

Then for all $L \in V^{*}$, there exists a unique element $\bar{u} \in V$ such that

$$
\frac{1}{2} a(\bar{u}, .)+\frac{1}{2} a(., \bar{u})=L .
$$

Moreover, if a is symmetric, then $\bar{u}$ is characterized by the property

$$
a(\bar{u}, .)=L
$$

Proof. In fact it suffices to set $J(v):=\frac{1}{2} a(v, v)-L(v)$. Since $J^{\prime}(u)=\frac{1}{2} a(u,)+.a(., u)-L$, one has for all $u, v \in V$

$$
<J^{\prime}(u)-J^{\prime}(v), u-v>=a(u-v, u-v),
$$

and since $a$ is coercive, then by Proposition 3.11, $J$ is 2 -strongly convex. Using Theorem 4.2 we get a unique $\bar{u} \in V$ such that $\frac{1}{2} a(\bar{u},)+.\frac{1}{2} a(., \bar{u})=L$ and when $a$ is symmetric we deduce

$$
a(\bar{u}, .)=L .
$$


4.2. Application to p-Laplace problem. To end this paper, we give an important application of our results.

Let $V:=W_{0}^{1, p}(\Omega)$ equipped with the norm $\|u\|^{p}=\sum_{i=1}^{N} \int_{\Omega}\left|\frac{\partial u(x)}{\partial x_{i}}\right|^{p} d x$, where $2 \leq p<+\infty$ and $\Omega \subset \mathbb{R}^{N}$ be an open, bounded and regular set. We are going to prove that the following boundary value problem

$$
(P) \quad\left\{\begin{array}{cc}
-\Delta_{p} u=f, & \text { on } \Omega, \\
u=0, & \text { on } \partial \Omega
\end{array}\right.
$$

where $\Delta_{p} u=\sum_{i=1}^{N} \frac{\partial}{\partial x_{i}}\left(\left|\frac{\partial u}{\partial x_{i}}\right|^{p-2} \frac{\partial u}{\partial x_{i}}\right)$, has an unique weak solution, which is equivalent to minimize the function, $J: V \rightarrow \mathbb{R} \cup\{+\infty\}$ defined by

$$
J(u)=\frac{1}{p} \sum_{i=1}^{N} \int_{\Omega}\left|\frac{\partial u(x)}{\partial x_{i}}\right|^{p} d x-\int_{\Omega} f(x) u(x) d x,
$$

where $f \in L^{q}(\Omega)$ with $\frac{1}{p}+\frac{1}{q}=1$. To this end, the following proposition is needed.

Proposition 4.5. The following assertions hold:

a) Let $1<p<+\infty$, then the function $J$ is Gâteaux differentiable on $V$ and for any $u, v$ in $V$, one has

$$
<J^{\prime}(u), v>=\sum_{i=1}^{N} \int_{\Omega}\left|\frac{\partial u(x)}{\partial x_{i}}\right|^{p-2} \frac{\partial u(x)}{\partial x_{i}} \frac{\partial v(x)}{\partial x_{i}} d x-\int_{\Omega} f(x) v(x) d x .
$$

b) Let $2 \leq p<+\infty$, then the function $J$ is p-strongly convex.

c) The boundary value problem $(P)$ has a unique weak solution $u \in V$.

Proof. a)Set for $t>0, h_{i}(t):=\left|\frac{\partial u(x)}{\partial x_{i}}+t \frac{\partial v(x)}{\partial x_{i}}\right|^{p}$ with $u, v$ in $\mathrm{V}$. We have $h_{i}^{\prime}(0)=p\left|\frac{\partial u(x)}{\partial x_{i}}\right|^{p-2}$ $\frac{\partial u(x)}{\partial x_{i}} \cdot \frac{\partial v(x)}{\partial x_{i}}$. Since the function, $r \rightarrow r^{p}$ from $\mathbb{R}^{+}$into $\mathbb{R}^{+}$is convex for $p \geq 1$ then for any $0<t<1$

Thus

$$
\left|\frac{\partial u(x)}{\partial x_{i}}+t \frac{\partial v(x)}{\partial x_{i}}\right|^{p} \leq(1-t)\left|\frac{\partial u(x)}{\partial x_{i}}\right|^{p}+t\left|\frac{\partial u(x)}{\partial x_{i}}+\frac{\partial v(x)}{\partial x_{i}}\right|^{p}
$$

$$
\frac{h_{i}(t)-h_{i}(0)}{t} \leq\left|\frac{\partial u(x)}{\partial x_{i}}+\frac{\partial v(x)}{\partial x_{i}}\right|^{p} .
$$

Since the right-hand side is in $L^{1}(\Omega)$, then by Lebesgue's dominated convergence theorem, we get

$$
<J^{\prime}(u), v>=\frac{1}{p} \sum_{i=1}^{N} \int_{\Omega} \lim _{t \rightarrow 0} \frac{h_{i}(t)-h_{i}(0)}{t} d x-\int_{\Omega} f(x) v(x) .
$$

Hence

$$
<J^{\prime}(u), v>=\sum_{i=1}^{N} \int_{\Omega}\left|\frac{\partial u(x)}{\partial x_{i}}\right|^{p-2} \frac{\partial u(x)}{\partial x_{i}} \frac{\partial v(x)}{\partial x_{i}} d x-\int_{\Omega} f(x) v(x) d x .
$$

b)Let $2 \leq p<+\infty$ and $u, v \in V$. One has by Clarkson's inequality (see p. 95 [6])

$$
\left\|\frac{1}{2}\left(\frac{\partial u}{\partial x_{i}}+\frac{\partial v}{\partial x_{i}}\right)\right\|_{L^{p}}^{p}+\left\|\frac{1}{2}\left(\frac{\partial u}{\partial x_{i}}-\frac{\partial v}{\partial x_{i}}\right)\right\|_{L^{p}}^{p} \leq \frac{1}{2}\left(\left\|\frac{\partial u}{\partial x_{i}}\right\|_{L^{p}}^{p}+\left\|\frac{\partial v}{\partial x_{i}}\right\|_{L^{p}}^{p}\right),
$$

for any $1 \leq i \leq N$. Therefore

$$
J\left(\frac{u+v}{2}\right) \leq \frac{1}{2}(J(u)+J(v))-c\|u-v\|^{p},
$$


where $c=\frac{1}{2^{p}}$. Since $J$ is l.s.c. then by Proposition 1.4 we may deduce that $J$ is p-strongly convex.

c) The assertions a) and b) allow us to deduce that $\mathrm{J}$ is p-strongly convex and Gâteaux differentiable for $2 \leq p<+\infty$. Therefore by Theorem 4.2 there exists $c>0$ such that for any $v \in V$

$$
c\|u-v\|^{p} \leq f(v)-f(u) .
$$

Which is equivalent to $0 \in \partial_{(p, c)} J(u)$ and since $\mathrm{J}$ is Gâteaux differentiable we may conclude that $J^{\prime}(u)=0$, what means

$$
\left\{\begin{array}{l}
\sum_{i=1}^{N} \int_{\Omega}\left|\frac{\partial u(x)}{\partial x_{i}}\right|^{p-2} \frac{\partial u(x)}{\partial x_{i}} \frac{\partial v(x)}{\partial x_{i}} d x=\int_{\Omega} f(x) v(x) d x, \quad \forall v \in V \\
u \in V
\end{array}\right.
$$

Or equivalently,

$$
\left\{\begin{array}{l}
-\sum_{i=1}^{N} \int_{\Omega} \frac{\partial}{\partial x_{i}}\left(\left|\frac{\partial u(x)}{\partial x_{i}}\right|^{p-2} \frac{\partial u(x)}{\partial x_{i}}\right) v d x=\int_{\Omega} f(x) v(x) d x, \quad \forall v \in V \\
u=0 \text { on } \partial \Omega
\end{array}\right.
$$

Indeed this boundary value problem is the nonlinear Laplacian so-called p-Laplacian which is Dirichlet problem. In particular $\mathrm{u}$ is a weak solution of the boundary value problem

$$
\left\{\begin{array}{cc}
-\Delta_{p} u=f, & \text { on } \Omega, \\
u=0, & \text { on } \partial \Omega,
\end{array}\right.
$$

Corollary 4.6. Let $2 \leq p<+\infty$. Then there exists $c>0$ such that for any $u, v \in V$

$\sum_{i=1}^{N} \int_{\Omega}\left(\left|\frac{\partial u(x)}{\partial x_{i}}\right|^{p-2} \frac{\partial u(x)}{\partial x_{i}}-\left|\frac{\partial v(x)}{\partial x_{i}}\right|^{p-2} \frac{\partial v(x)}{\partial x_{i}}\right)\left(\frac{\partial u(x)}{\partial x_{i}}-\frac{\partial v(x)}{\partial x_{i}}\right) d x \geq c \sum_{i=1}^{N} \int_{\Omega}\left|\frac{\partial u(x)}{\partial x_{i}}-\frac{\partial v(x)}{\partial x_{i}}\right|^{p} d x$.

Proof. This fact is a consequence of Proposition 4.5 and Corollary 3.11.

\section{REFERENCES}

[1] G. Allaire, Analyse Numérique et Optimisation, Editions de l'école polytechnique, Palaiseau 2012.

[2] A. Allali and T. Amahroq, On the openness and regularity of $\gamma$-paraconvex, Control Cybern. 26 (1997) 87-92.

[3] T. Amahroq and A.Taa, Optimality conditions for multiobjective optimization problems with $\gamma$-paraconvex data, Stud. Math. 124 (1997) 239-248. http://matwbn.icm.edu.pl/ksiazki/sm/sm124/sm12433.pdf.

[4] D. Aussel, J.-N. Corvellec and M. Lassonde, Mean value property and subdifferential criteria for lower semicontinuous functions, Trans. Amer. Math. Soc. 347 (1995) 4147-4161. https://doi .org/10.2307/2155218

[5] J. M. Borwein, A note on $\varepsilon$-subgradients and maximal monotonicity, Pac. J. Math. 103 (1982) $307-314$.

[6] H. Brezis, Analyse fonctionnelle. Masson, Paris, 1983.

[7] F. H. Clarke, Optimization and nonsmooth analysis, Wiley-Interscience, New York, 1983.

[8] R. Correa, A. Jofré and L. Thibault, Characterization of lower semicontinous convex functions, Proc. Amer. Math. Soc. 116 (1992) 61-72. https://doi.org/10.2307/2159295.

[9] R. Correa, A. Jofré and L. Thibault, Subdifferential monotonicity as characterization of convex functions, Numerical Funct. Anal. Optim. 15 (1994) 531-535. https://doi.org/10.1080/01630569408816579.

[10] A. D. Ioffe, Approximate subdifferentials and applications, II and III, Mathematika 33 (1986) 111-128; 36 (1989) 1-38. https://doi.org/10.1112/S0025579300013930. 
[11] A. Jourani, Subdifferentiability and subdifferential monotonicity of $\gamma$-paraconvex functions, Control Cybern. 25 (1996) 721-737. http://jourani.perso.math.cnrs.fr/jourani-subdiff.pdf

[12] L. McLinden, An application of Ekeland's theorem to minimax problems, Nonlinear Anal. 6 (1982) 189-196. https://doi.org/10.1016/0362-546X (82)90087-6.

[13] B. S. Mordukhovich, Metric approximations and necessary optimality conditions for general classes of non smooth extremal problems, Soviet Math. Dokl. 22 (1980) 526-530. http://www . mathnet.ru/php/archive. phtml?wshow=paper\&jrnid=dan\&paperid=43962\&option_lang=eng.

[14] R. R. Phelps, Convex functions, monotone operators and differentiability, Lecture Notes in Math., vol. 1364, Springer-Verlag, Berlin, 1993.

[15] R. A. Poliquin, Subgradient monotonicity and convex functions, Nonlinear Anal. Theory Methods Appl. 14 (1990) 305-317. https://doi.org/10.1016/0362-546X (90) 90167-F.

[16] B. T. Polyak, Existence theorems and convergence of minimizing sequences in extremum problems with restrictions, Sov. Math. Dokl. 7 (1966) 72-75.

[17] R. T. Rockafellar, Princeton University Press, 1970.

[18] R. T. Rockafellar, Directionally lipschitzian functions and subdifferential calculus, Proc. London Math. Soc. 39 (1979) 331-355. https://sites.math.washington.edu/ rtr/papers/ rtr080-DirectionallyLipFns.pdf.

[19] R. T. Rockafellar, Generalized directional derivatives and subgradients of nonconvex functions, Can. J. Math. 32 (1980) 257-280.

[20] S. Rolewicz, On $\gamma$-paraconvex multifunctions, Math. Japonica. 24 (1979) 293-300. http://matwbn.icm. edu.pl/ksiazki/cc/cc34/cc34316.pdf.

[21] S. Rolewicz, On paraconvex multifunctions, Oper. Res. Verfahren. 31 (1979) 539-546. https://zbmath. org/?q=an: 0403.49021.

[22] S. Rolewicz, Paraconvex analysis, Control and Cybernetics. 34 (2005) 951-965. http://matwbn.icm.edu . $\mathrm{pl} / \mathrm{ksiazki} / \mathrm{cc} / \mathrm{cc} 34 / \mathrm{cc} 34316$.pdf.

[23] L. Thibault, A note on the Zagrodny mean value theorem, Optimization. 35 (1996) 127-130. https: //doi.org/10.1080/02331939508844134.

[24] D. Zagrodny, Approximate mean value theorem for upper subderivatives, Nonlinear Anal. Theory Meth. Appl. 12 (1988) 1413-1438. https://doi.org/10.1016/0362-546X (88)90088-0. 